\title{
The Interaction Test of Binary Mixtures of Endocrine-Disrupting Chemicals Using In Vitro Bioassays
}

\author{
Qianqian Tang, ${ }^{1}$ Bingli Lei $\mathbb{D}^{1},{ }^{1}$ Yun Liu, ${ }^{2}$ Xiaolan Zhang, ${ }^{1}$ Qian Liu, ${ }^{1}$ and Su Sun ${ }^{1}$ \\ ${ }^{1}$ Institute of Environmental Pollution and Health, School of Environmental and Chemical Engineering, Shanghai University, \\ Shanghai 200444, China \\ ${ }^{2}$ South China Institute of Environmental Sciences, MEP, 7 West Street, Yuancun, Tianhe District, Guangzhou 510655, China \\ Correspondence should be addressed to Bingli Lei; leibingli@126.com
}

Received 9 August 2019; Revised 10 January 2020; Accepted 3 June 2020; Published 22 June 2020

Guest Editor: Lisa Yu

Copyright (c) 2020 Qianqian Tang et al. This is an open access article distributed under the Creative Commons Attribution License, which permits unrestricted use, distribution, and reproduction in any medium, provided the original work is properly cited.

Typical environmental endocrine-disrupting chemicals (EDCs) such as estradiol valerate (EV), diethylstilbestrol (DES), di-2ethylhexyl phthalate (DEHP), mono-2-ethylhexyl phthalate (MEHP), and bisphenol A (BPA) have a strong reproductive and developmental toxicity at low concentrations. However, information on their joint toxicity is scarce. In this study, we evaluated the combined effects of EV and other four EDCs (DES, DEHP, MEHP, and BPA) on the human breast MCF-7 cells by detecting the cell proliferation, intracellular reactive oxygen species (ROS) levels, and estrogen receptor alpha (ER $\alpha$ ) protein expression using equal concentration ratio method. The results showed that, after exposure for 24,48 , and $72 \mathrm{~h}$, single EV, DES, and BPA can promote the proliferation of MCF-7 human breast cancer cells, and EV has the strongest effect in inducing cell proliferation. DEHP and MEHP cannot induce MCF-7 cell proliferation for all exposure time, while cell proliferation induced by EV was significantly attenuated by DES, BPA, DEHP, and MEHP when they mixed with EV. For intracellular ROS, single EV, BPA, DES, DEHP, and MEHP elevated intracellular ROS levels for different exposure time. Similar to the cell proliferation, DES and BPA decreased intracellular ROS levels induced by EV when they mixed with EV for $24 \mathrm{~h}$. EV, DES, and BPA exposed alone or combined with EV upregulated the ER $\alpha$ protein expression. However, DEHP and MEHP exposed alone or combined with EV had no effect on ER $\alpha$ protein expression, indicating that DEHP or MEHP could attenuate ER $\alpha$ protein expression upregulated by EV. These results showed that the joint toxicity of binary mixtures of EV and other EDCs do not interact in a synergistic fashion in inducing cell proliferation, intracellular ROS levels, and ER $\alpha$ protein expression. These findings have important implications in the human risk assessments of EV mixed with other EDCs in the environment.

\section{Introduction}

Environmental endocrine-disrupting chemicals (EDCs) such as estradiol valerate (EV), diethylstilbestrol (DES), bisphenol A (BPA), diethylhexyl phthalate (DEHP), and mono-2-ethylhexyl phthalate (MEHP) bring up prevalent attention because they have a strong reproductive and developmental toxicity on human and various animal species at low doses $[1,2]$.

$\mathrm{EV}$ is one of the most common sources of free $\mathrm{E} 2$ in hormone replacement therapy [3]. In animal farming, EV is mainly applied as a growth promoter [4] and for developing a single-sex population of fish in aquaculture $[5,6]$. EV may act as an endocrine disruptor and adversely affect reproductive outcome [7]. EV has been detected in natural waters in China, and its concentration range is approximately $1-10 \mathrm{ng} / \mathrm{L}[8,9]$. DES was the first synthetic estrogen originally used for clinical therapy to prevent miscarriages. However, exposure to DES can cause adverse effects on male and female reproductive tracts in humans, even causing developmental anomalies in subsequent generations [10]. Though the use of DES has been banned in humans, it is still employed in livestock and aquaculture operations as a growth promoter or to produce monosex (female) populations of fish in some parts of the world [11]. DES is also quite potent in aquatic organisms such as fish and may be comparable to, or even exceed, that of $17 \alpha$-ethinylestradiol (EE2) with a predicted no-effect concentration (PNEC) in 
fish lower than $1 \mathrm{ng} / \mathrm{L}$ [12]. Detectable concentrations of DES in surface water and effluents from different locations have been reported to range from slightly below 1 to greater than $10 \mathrm{ng} / \mathrm{L}$ [13-16]. BPA, as one of the high-volume chemicals produced worldwide, has wide applications in organic synthesis industry as raw material in the manufacture of plastics and epoxy resins [17]. BPA has been widely detected in various environmental media, foodstuffs, and human samples [17]. Exposure to BPA has shown lowdose effects including disturbed mammary gland development, changes in normal behavioral development, and changes in obesity associated parameters in rodents [17]. DEHP and MEHP belong to phthalates, and MEHP is metabolite of DEHP. DEHP is used in raw materials of clothing, food packaging, building, and children's products [18]. DEHP pollution has raised public concern, especially after the plasticizer incident in Taiwan in May 2011. High levels of DEHP are detected in municipal domestic waste, soil, rivers, fruits, vegetables, plastic-wrapped food, and medical devices to which people are closely related. A recent study indicated that the concentration of DEHP was $78 \mu \mathrm{g} / \mathrm{L}$ in Bohai Sea (in Tianjin, China), and MEHP was also found at a relatively high concentration [19]. DEHP has been reported to have carcinogenic, mutagenic, and hepatotoxic effects [20]. These compounds can affect the reproductive health of organisms at low concentrations. However, the estrogen activity and reproductive toxicity have been extensively studied mainly based on individual EDCs. Few studies have addressed the joint effects of mixtures of these compounds on estrogen-dependent responses. Estrogen activity of these individual EDCs is relatively weak; even DEHP and MEHP present antiestrogenic activity [21, 22]; however, the "real-life" mixtures of EDCs may carry significant biological potency [18]. Therefore, the joint toxicity of complex EDCs should not be ignored and potential interactions of weakly anti-or estrogenic substances could have important implications for public health.

The estrogen-sensitive tumor cell proliferation is an ideal method for evaluating estrogenic activity of compounds [23]. Occurrence of reactive oxygen species (ROS) in estrogensensitive cells has been a common response of chemical estrogenic activity [24]. For environmental estrogen, it is widely accepted that a moderate increase of ROS can stimulate proliferation of estrogen-sensitive tumor cells $[25,26]$. Besides, environmental estrogen can induce estrogen-sensitive cell proliferation and may increase mitochondrial ROS production by estrogen receptor alpha/ $\beta$ eta $(\mathrm{ER} \alpha / \beta)$ pathway [27]. In this study, we evaluate the effects of single EV, DES, BPA, DEHP, and MEHP on proliferation of MCF-7 cells to assess their estrogen activities. Among these EDCs, EV induced the highest MCF-7 cell proliferation. Its estrogenic potency is similar to estradiol (E2). To evaluate influence of other EDCs on cellular biological effects induced by EV, we further assess joint toxicity of the binary mixtures of EV and other four EDCs by measuring the cell proliferation, ROS generation, and ER $\alpha$ expression in MCF-7 cells. In order to simplify the joint toxicity experimental method, the equal concentration ratio mixing method was widely recommended in joint toxicity mechanism research of binary mixtures
$[28,29]$. In this study, we also used equal concentration ratio mixing method to study joint toxicity of binary mixtures of EV and the other four EDCs. The results can provide a comprehensive understanding of binary mixture of EV and other EDCs toxicity effects.

\section{Materials and Methods}

2.1. Materials. E2 (estradiol), DES (diethylstilbestrol), EV (estradiol valerate), BPA (bisphenol A), DEHP (diethylhexyl phthalate), and MEHP (mono-2-ethylhexyl phthalate) were purchased from Sigma (Saint Louis, MO, USA) and were of the highest grade commercially available.

The stock solutions of all target chemicals were prepared in dimethyl sulfoxide (DMSO, 99.5\%, AMRESCO, PA, USA) and were stored at $-20^{\circ} \mathrm{C}$. Cell Counting Kit-8 (CCK-8) was purchased from Dojindo (Kumamoto, Japan). $2^{\prime}, 7^{\prime}$ Dichlorodihydrofluorescein diacetate (DCFH-DA) and tertbutyl hydroperoxide (tBHP) were purchased from Sigma (Saint Louis, MO, USA). MCF-7 breast cancer cell line was purchased from American Tissue Culture Collection (Rockville, MD, USA). All other reagents used were analytical grade chemicals from Sigma (Saint Louis, MO, USA), if not otherwise stated.

2.2. Maintenance and Treatments of MCF-7 Cells. In our previous study, cell maintenance and treatment of MCF-7 cells are described in detail [30]. The specific process is that stock cultures of MCF-7 cells were maintained in regular RPMI 1640 medium (HyClone, Logan, UT, USA) containing $10 \%$ fetal bovine serum (FBS) (Gibco, Grand Island, NY) and supplemented with $100 \mathrm{U} / \mathrm{mL}$ penicillin and $100 \mathrm{U} / \mathrm{mL}$ streptomycin (Invitrogen, Burlington, ON, Canada) in a humidified atmosphere at $37^{\circ} \mathrm{C}$ with $5 \% \mathrm{CO}_{2}$. The medium was changed every $2-3 \mathrm{~d}$.

The exponential growth phase cells were used for the experiments. Before each experiment, cells were starved in steroid-free (SF) medium for $24 \mathrm{~h}$ to minimize the basal hormonal levels during assays and allow cell adhesion. SF medium consists of phenol red-free RPMI 1640 (HyClone, Logan, UT, USA) and 5\% charcoal-stripped FBS (Biological Industries, Israel) supplemented with $100 \mathrm{U} / \mathrm{mL}$ streptomycin/penicillin. $0.1 \%$ DMSO was used as the solvent control in each experiment. For ROS experiment, $400 \mu \mathrm{M}$ tBHP was used as the positive control. In this study, we followed the methods described by Lei et al. 2017 [30]. The tBHP significantly increased intracellular ROS production and the ROS level (compared to control $\times 100$ ) of MCF-7 cells treated with $400 \mu \mathrm{M}$ tBHP was $280 \pm 35.6 \%$.

2.3. Cell Viability Assay. In order to study the proliferation effect of tested compounds on MCF-7 cells, CCK-8 was used to measure the viability of MCF-7 cells. The specific experiment methods were provided elsewhere $[23,30]$. Briefly, the treated cells were added with the CCK- 8 reagents $(10 \mu \mathrm{L}$ in $90 \mu \mathrm{L}$ phenol red-free RPMI 1640 per well), and optical density (OD) was read with a Multiskan MK3 plate reader (Thermo Electron Corporation, Waltham, MA, USA) at a 
wavelength of $490 \mathrm{~nm}$. The OD value can reflect the number of living cells indirectly, and cell viability (\% of control) was calculated according to the OD values of exposure group and control group.

For joint toxicity, the cells were exposed to binary mixtures of EV and the other four EDCs according to equal concentration ratio method. The corresponding cell viability was detected.

2.4. ROS Detection. Intracellular ROS were assayed using a fluorescent dye DCFH-DA. The specific experiment methods were provided elsewhere [30]. Briefly, the treated cells were incubated with $50 \mu \mathrm{L}$ DCFH-DA $\left(2 \times 10^{-4} \mathrm{M}\right)$ at $37^{\circ} \mathrm{C}$ for $30 \mathrm{~min}$ in the dark, and the fluorescence was evaluated under a fluorescence microscope (Olympus BX51; Olympus, Japan). The intensity of fluorescence was analyzed by Image-Pro Plus 6 .

For joint toxicity, the cells were exposed to binary mixtures of EV and the other four EDCs according to the equal concentration ratio method, and the corresponding ROS levels were detected according to the above method.

2.5. Western Blot. MCF-7 cells were collected after exposure to single E2, EV, DES, BPA, DEHP, DES $(1-100 \mathrm{nM})$, and $0.1 \%$ DMSO (negative control) for $24 \mathrm{~h}$. The specific experiment was described in our previous study [30]. Briefly, total protein samples of $\mathrm{ER} \alpha$ were obtained by mammalian protein extraction reagent (M-PER). Equal amounts $(20 \mu \mathrm{g})$ of protein samples were subjected to $10 \%$ sodium dodecyl sulfate-polyacrylamide gel electrophoresis (SDS-PAGE) and were transferred from the gels onto nitrocellulose membranes. The used specific monoclonal antibodies are anti-ER $\alpha(1: 1000)$ and anti-glyceraldehyde-3-phosphate dehydrogenase (GAPDH) $(1: 2000)$.

To study the joint effect of binary mixtures of EV and the other four EDCs on ER $\alpha$ protein expression, the cells were coexposed to binary mixtures of EV and the four EDCs according to the equal concentration ratio method for $24 \mathrm{~h}$. Thereafter, ER $\alpha$ protein samples were collected, and protein expression was analyzed.

2.6. Data Analysis. All results are expressed as the mean\pm standard deviation (SD). The differences between exposure groups and the negative control were tested using one-way analysis of variance with specific mean comparisons by Dunnett's test. Student's $t$-test was used to analyze the difference between two different exposure groups. Difference was considered significant at a $p$ value $<0.05$. For $\mathrm{E} 2$ and $\mathrm{EV}$, the concentration-response analyses were performed with fourparameter logistic curve regression analysis, and EC50 was calculated, which is the concentration that induces half of the maximum proliferation effect.

\section{Results}

3.1. The Effects of Tested Compounds on the Viability of MCF-7 Cells. The CCK-8 assay showed that E2 at all exposure concentrations and all exposure time significantly induced cell viability except for the lowest concentration at $24 \mathrm{~h}$ (Figure 1(a)). The increases of cell viability presented very well dose-dependent, for the concentration range of 0.0001-1000 nM, and time-dependent manner. The EC50 values of $\mathrm{E} 2$ for 24,48 , and $72 \mathrm{~h}$ were $0.20,0.16$, and $0.059 \mathrm{nM}$, respectively. The cell viability reached the maximum value at about $1 \mathrm{nM}$ and then reached a platform period. EV at all exposure concentrations and all exposure time significantly induced the viability of MCF-7 cells and presented a concentration-dependent manner at the concentration range of 0.0001-1000 nM (Figure 1(b)). Maximal induction of EV was caused by $100 \mathrm{nM}$ for $24 \mathrm{~h}$ and $1000 \mathrm{nM}$ for 48 and $72 \mathrm{~h}$, and the highest proliferation ratio reached about 2.8 times that of the negative control. The EC50 values for 24,48 , and $72 \mathrm{~h}$ were $6.02,0.40$, and $0.33 \mathrm{nM}$, respectively.

The single effect of DES or EV and their joint effect on the viability of MCF-7 cells are shown in Figure 2. The cell viability was significantly induced by DES for all exposure concentrations for different exposure periods. When the cells were exposed to single DES $(0-10000 \mathrm{nM})$, the cell viability increased from $100 \%$ to $200 \%$. The joint effect of DES and EV on the viability of MCF-7 cells is shown in Figure 2. DES addition significantly attenuated cell viability induced by EV for three exposure periods and all exposure concentrations except for exposure concentration of $10000 \mathrm{nM}$ at $24 \mathrm{~h}$. In this exposure concentration and exposure time, DES addition significantly increased cell viability induced by $\mathrm{EV}$. At $48 \mathrm{~h}$, the cell viability induced by binary mixtures of EV and DES was the lowest, and binary mixtures of EV and DES showed antagonistic effect on the viability of MCF-7.

The single effect of BPA or EV and their joint effect on the viability of MCF-7 cells are shown in Figures 3(a)$3(\mathrm{c})$, respectively. BPA, only at the two highest exposure concentrations of 1000 and $10000 \mathrm{nM}$, significantly induced cell viability for three exposure periods. BPA significantly attenuated cell viability induced by EV when BPA mixed with EV according to equal concentrations except for $10000 \mathrm{nM}$ for $24 \mathrm{~h}$. At the highest exposure concentration of $10000 \mathrm{nM}$ for 48 and $72 \mathrm{~h}$, the binary mixtures of BPA and EV significantly inhibited cell proliferation. In addition, the binary mixtures of EV and BPA showed antagonistic effect on the viability of MCF-7 cells at 48 and $72 \mathrm{~h}$.

The single effect of DEHP or EV and their joint effect on the viability of MCF-7 cells are shown in Figures 4(a)-4(c). DEHP cannot induce significant cell proliferation for three exposure periods. The binary mixtures of DEHP and EV induced significant cell viability; however, cell viability induced by the binary mixtures of DEHP and EV was significantly lower than that induced by single EV. This indicated that DEHP addition decreased cell viability induced by EV. Similarly, single MEHP or the binary mixtures of MEHP and EV cannot all induce significant cell viability for all exposure concentrations (Figure 5). This result indicated that MEHP addition completely inhibited cell viability induced by single EV. 


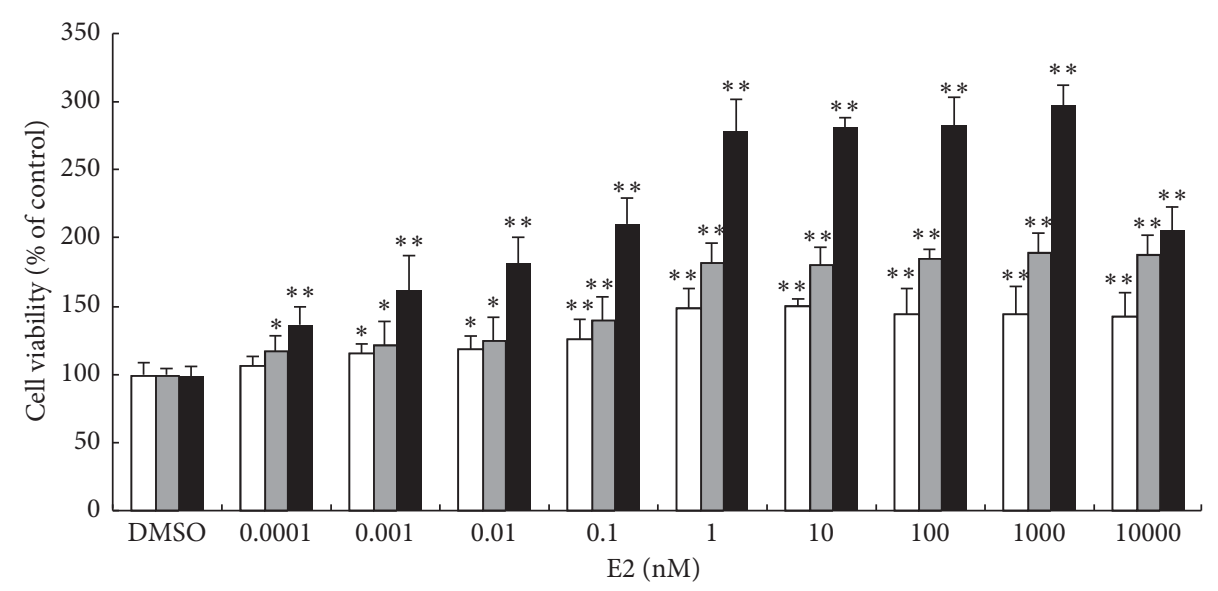

$$
\begin{aligned}
& \square 24 \mathrm{~h} \\
& \square 48 \mathrm{~h} \\
& \square 72 \mathrm{~h}
\end{aligned}
$$

(a)

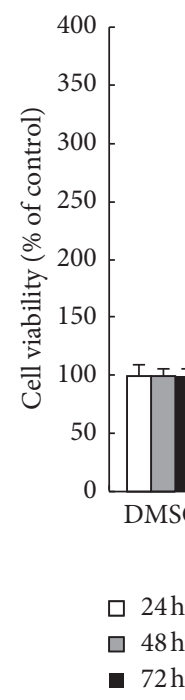

(b)

FIGURE 1: Effects of E2 (a) and EV (b) on the viability of MCF-7 cells. Data points represent mean \pm SD of three independent experiments. Asterisks show statistically significant differences with respect to corresponding negative DMSO $\left({ }^{*} p<0.05,{ }^{* *} p<0.01\right)$.

3.2. ROS Levels. As shown in Figure 6, the dose response for ROS formation is demonstrated with MCF-7 cells for individual chemicals alone or combined with EV. With the prolongation of exposure time, EV induced more ROS production. At $24 \mathrm{~h}, \mathrm{EV}$ induced the highest ROS generation in MCF-7 cells. The binary mixtures of EV and other EDCs presented different change trend in inducing intracellular ROS generation for different concentrations and different exposure time.

At $24 \mathrm{~h}$, the binary mixtures of EV and DES did not elevate intracellular ROS levels, while single EV significantly increased intracellular ROS levels, indicating that DES addition inhibited ROS generation induced by EV. At 1 or $3 \mathrm{~h}$, the binary mixtures of EV and DES increased intracellular ROS levels, but the ROS levels induced by EV and DES mixtures were lower than those induced by single EV or DES. Similarly, at $24 \mathrm{~h}$, the binary mixtures of EV and BPA cannot significantly elevate intracellular ROS levels. At 1 and
$3 \mathrm{~h}, \mathrm{EV}$ and BPA binary mixtures significantly increased intracellular ROS, and ROS levels induced by them were higher than those induced by single EV. The results indicated that BPA addition increased ROS levels induced by single EV for 1 and $3 \mathrm{~h}$ and decreased intracellular ROS levels induced by single EV for $24 \mathrm{~h}$. Similarly, at 1 and $3 \mathrm{~h}$, EV and DEHP mixtures significantly increased intracellular ROS levels, but the ROS levels induced by them had no significant difference between EV and DEHP binary mixtures and single EV. From the above results, at $24 \mathrm{~h}$, antigenic effects of binary mixtures of EV and DES or BPA can be found in inducing intracellular ROS generation.

3.3. The Effects of Target Compounds on Protein Expression of $E R \alpha$ in MCF-7 Cells. To detect the effects of these EDCs on $\mathrm{ER} \alpha$ protein expression in MCF-7 cells, ER $\alpha$ protein 


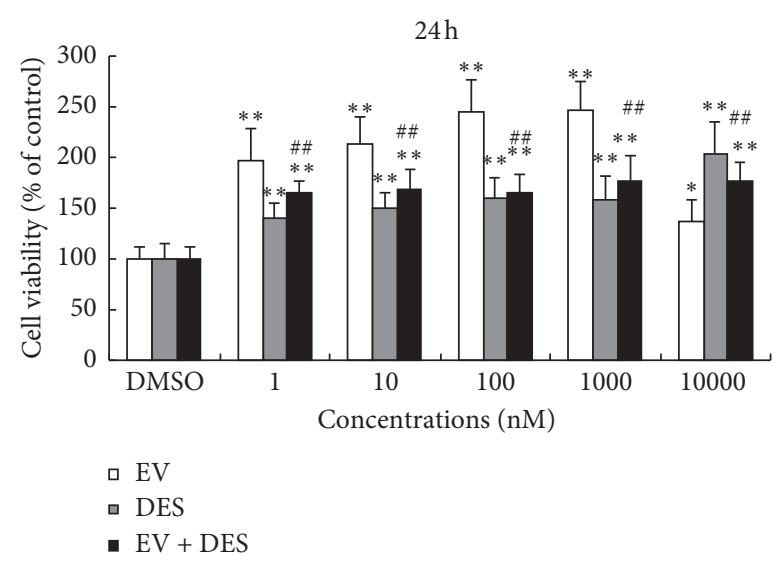

(a)

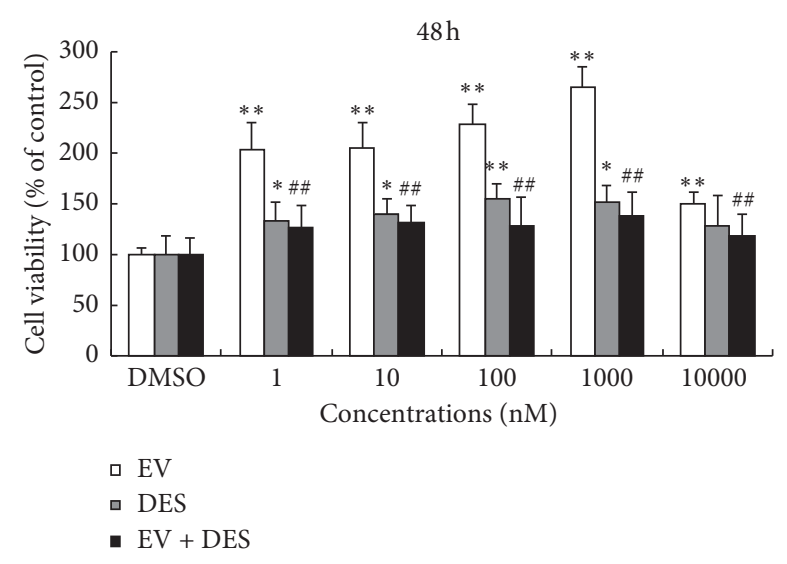

(b)

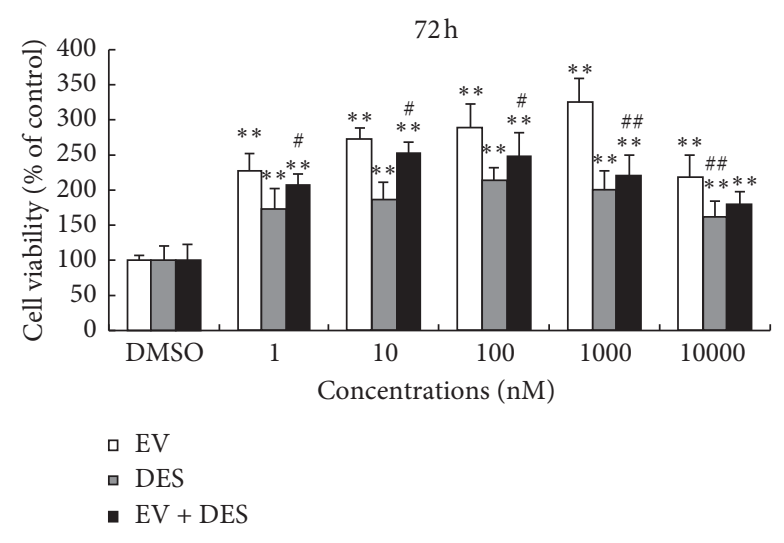

(c)

FIgURE 2: The binary joint effect of EV and DES on cell viability of MCF-7 cells. Exposure time: (a) $24 \mathrm{~h}$; (b) 48 h; (c) $72 \mathrm{~h}$. Data points represent mean $\pm \mathrm{SD}$ of three independent experiments. ${ }^{*} p<0.05,{ }^{* *} p<0.01$ compared with DMSO; ${ }^{\#} p<0.05$, ${ }^{\# \#} p<0.01$, compared with EV treatment.

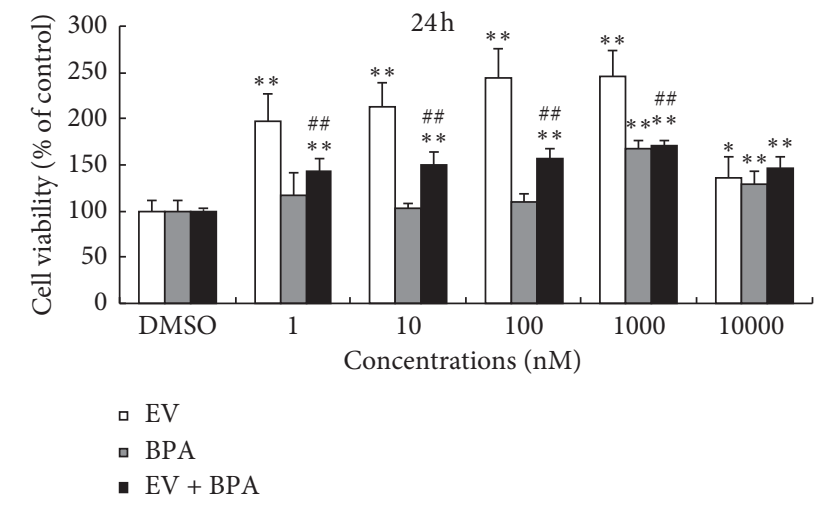

(a)

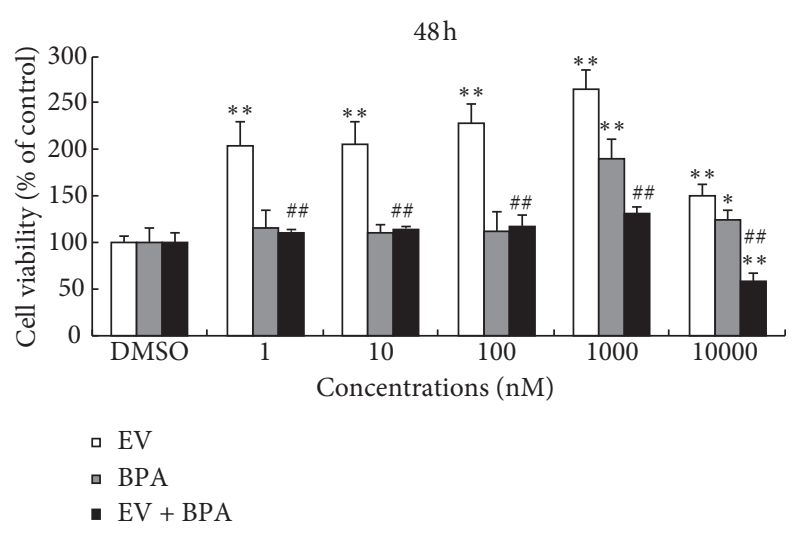

(b)

FIgURE 3: Continued. 


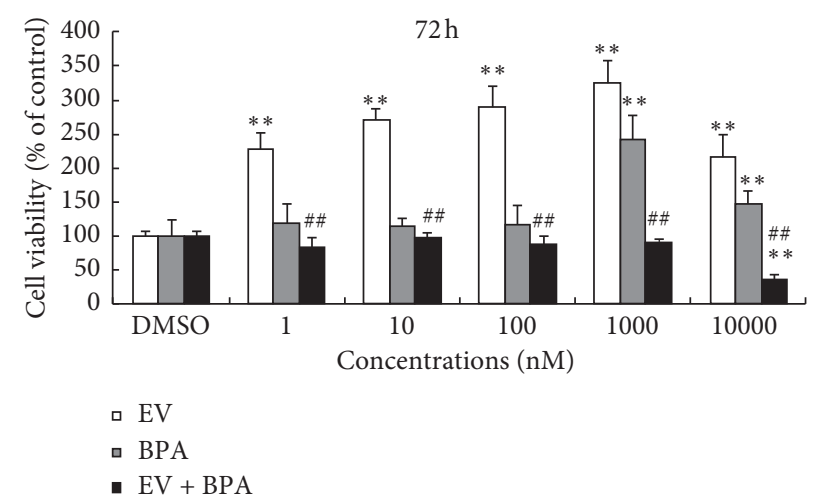

(c)

FIgURE 3: The binary joint effect of EV and BPA on cell viability of MCF-7 cells. Exposure time: (a) $24 \mathrm{~h}$; (b) $48 \mathrm{~h}$; (c) $72 \mathrm{~h}$. Data points represent mean $\pm \mathrm{SD}$ of three independent experiments. ${ }^{*} p<0.05,{ }^{* *} p<0.01$, compared with DMSO; ${ }^{\# \#} p<0.01$, compared with EV treatment.

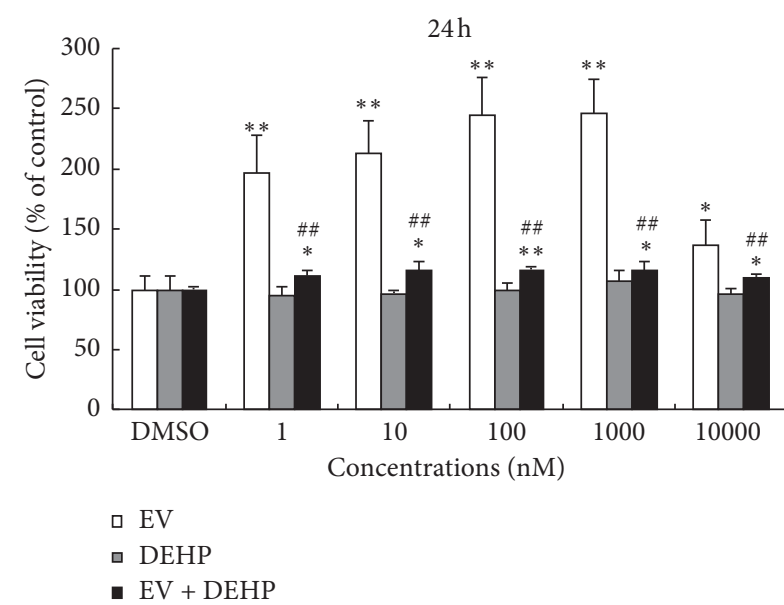

(a)

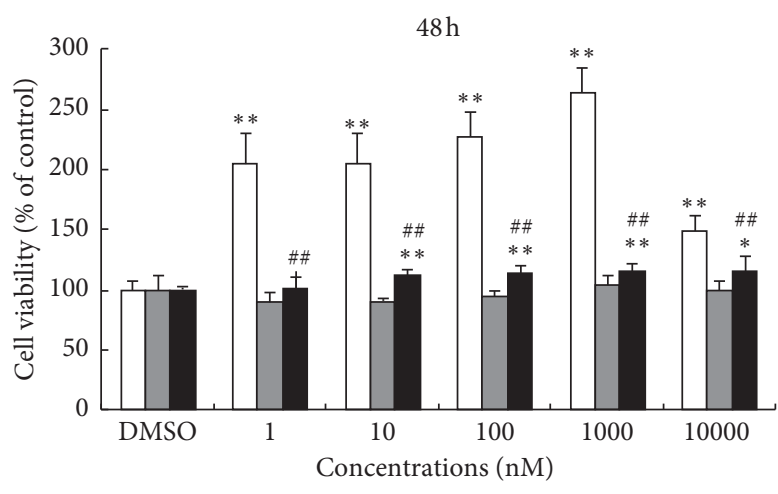

$\square \mathrm{EV}$

$\square$ DEHP

- $\mathrm{EV}+\mathrm{DEHP}$

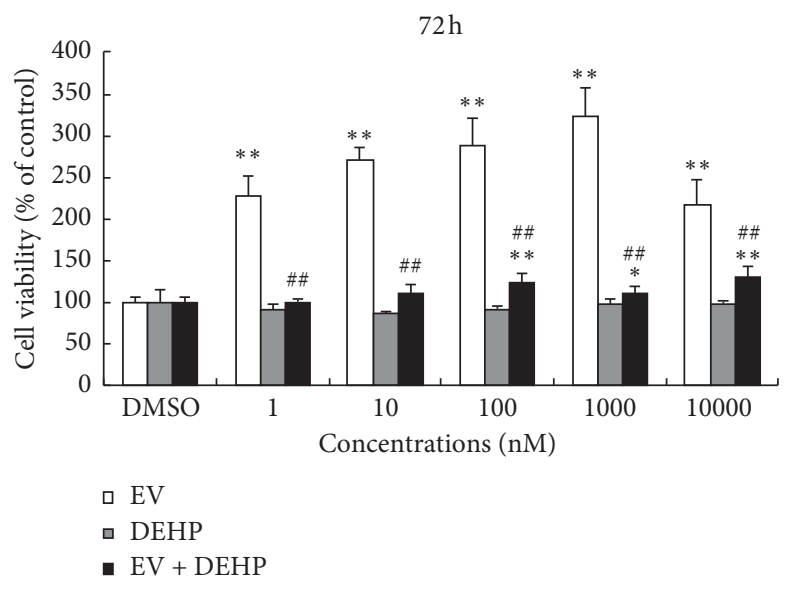

(b)

(c)

Figure 4: The binary joint effects of EV and DEHP on cell viability of MCF-7 cells. Exposure time: (a) $24 \mathrm{~h}$; (b) $48 \mathrm{~h}$; (c) $72 \mathrm{~h}$. Data points represent mean $\pm \mathrm{SD}$ of three independent experiments with six replications. ${ }^{*} p<0.05,{ }^{* *} p<0.01$, compared with DMSO; ${ }^{\# \#} p<0.01$, compared with EV treatment. 


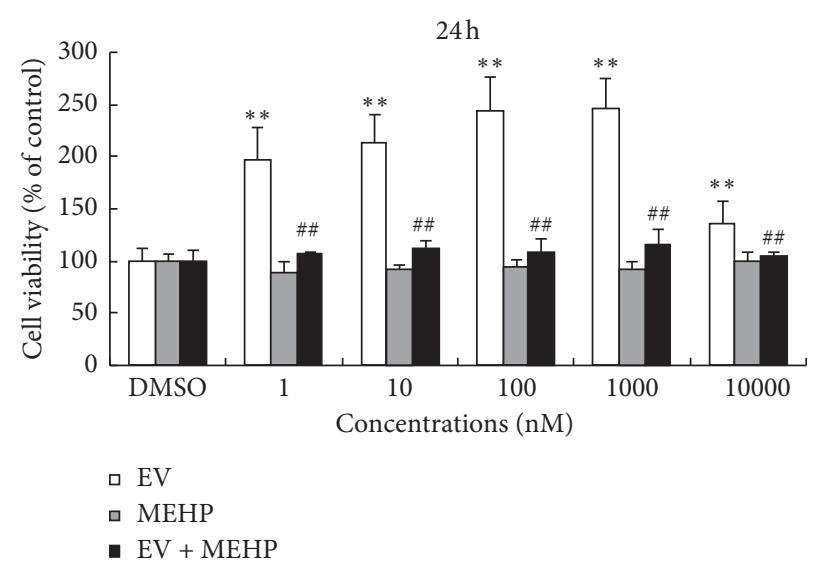

(a)

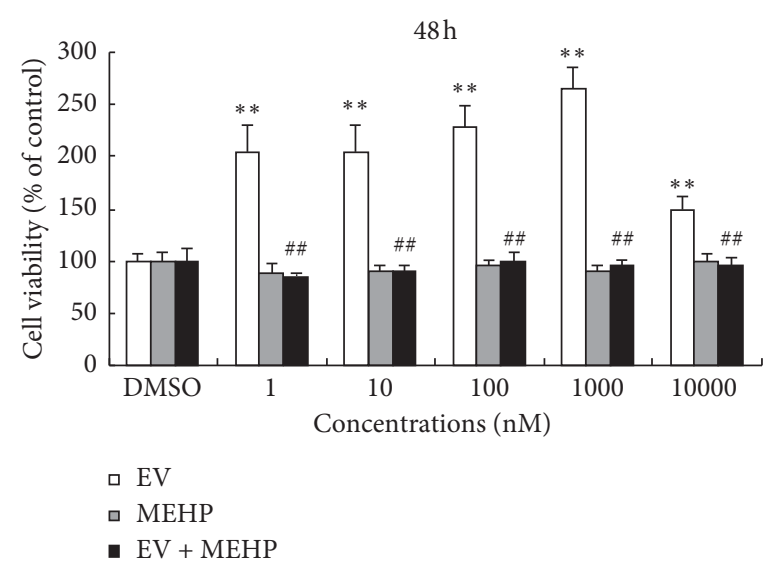

(b)

FIGURE 5: The binary joint effects of EV and MEHP on cell viability of MCF-7 cells. Exposure time: (a) $24 \mathrm{~h}$; (b) $48 \mathrm{~h}$. Data points represent mean \pm SD of three independent experiments with six replications. ${ }^{*} p<0.05,{ }^{* *} p<0.01$, compared with DMSO; ${ }^{\# \#} p<0.01$, compared with EV treatment.

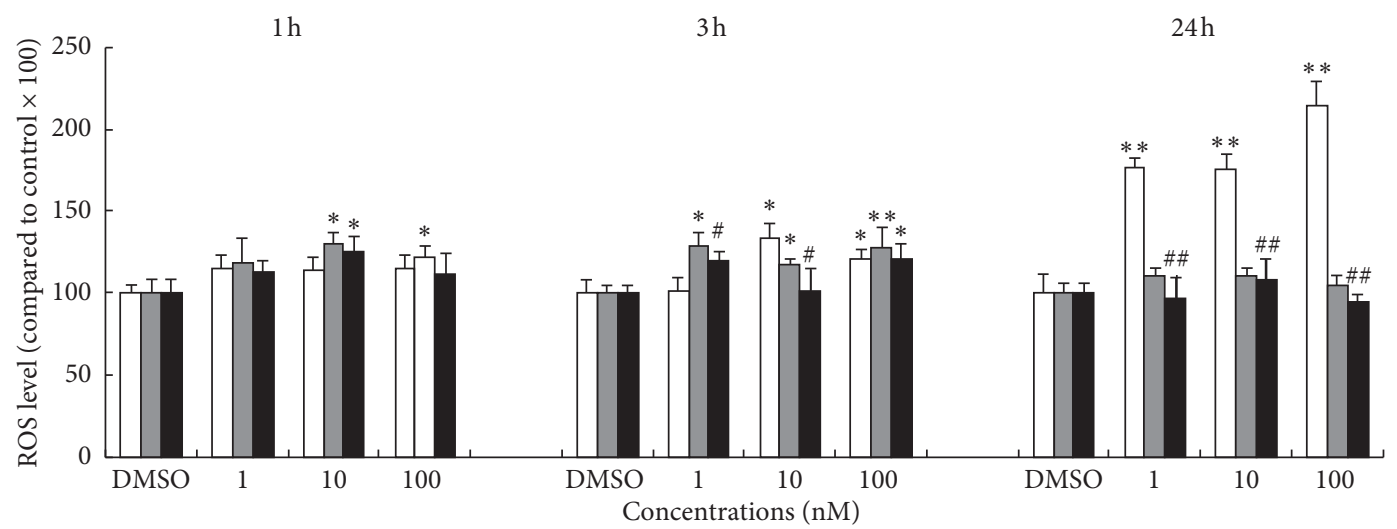

口 EV

- DES

- $\mathrm{EV}+\mathrm{DES}$

(a)

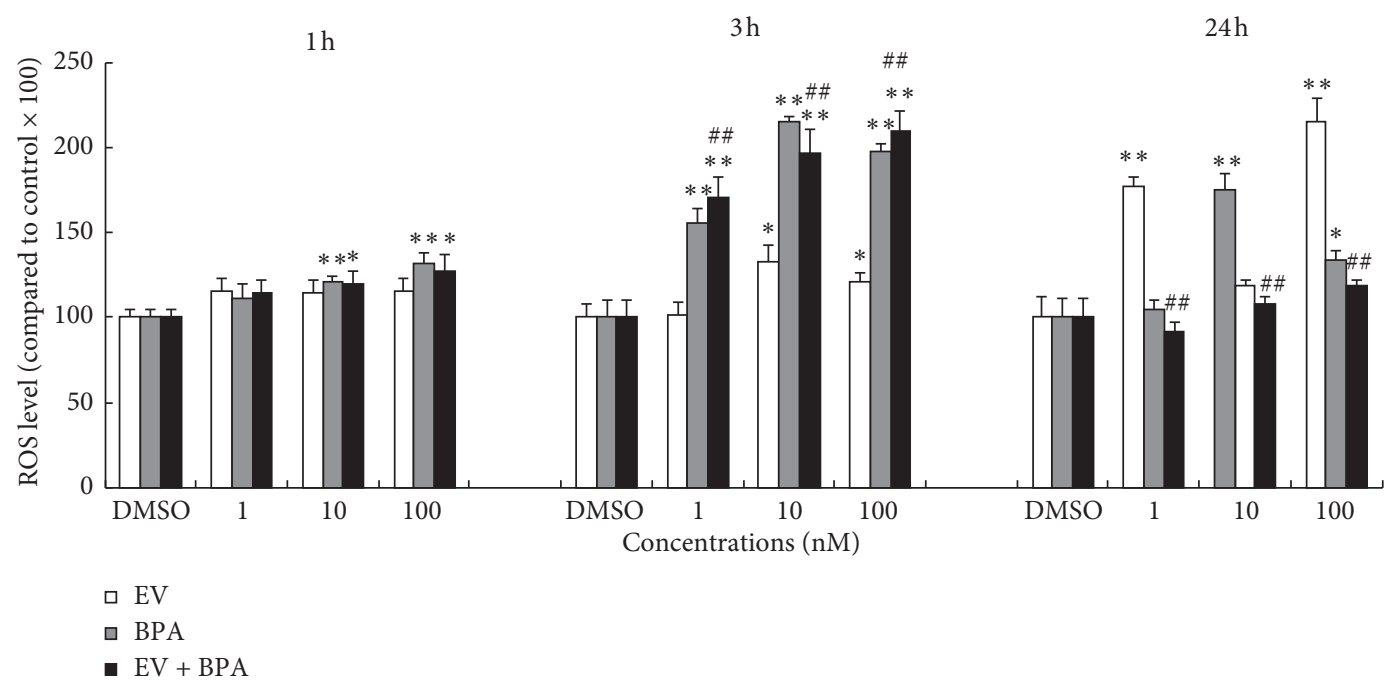

(b)

FIgURE 6: Continued. 


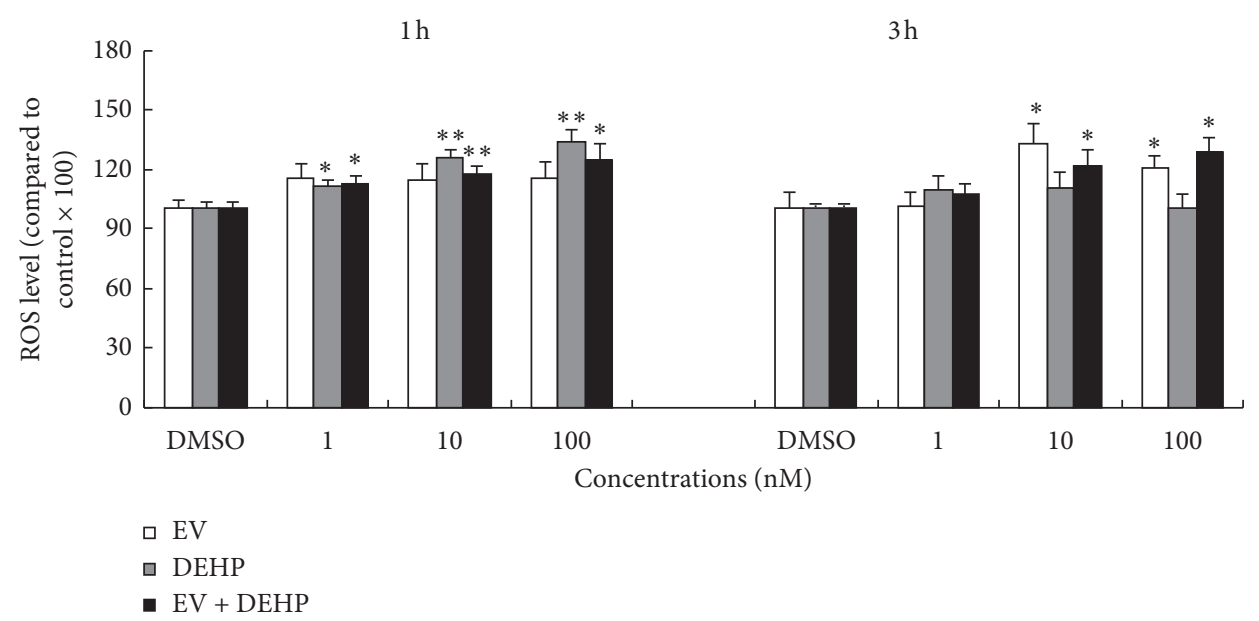

(c)

FIGURE 6: The binary joint effects of tested compounds and EV on the intracellular ROS generation in MCF-7 cells. A binary joint system of (a) DES and BPA; (b) BPA and EV; (c) DEHP and EV. Data points represent mean \pm SD of three independent experiments. ${ }^{*} p<0.05$, ${ }^{* *} p<0.01$, compared with DMSO; ${ }^{\#} p<0.05$, ${ }^{\# \#} p<0.01$, compared with EV treatment.

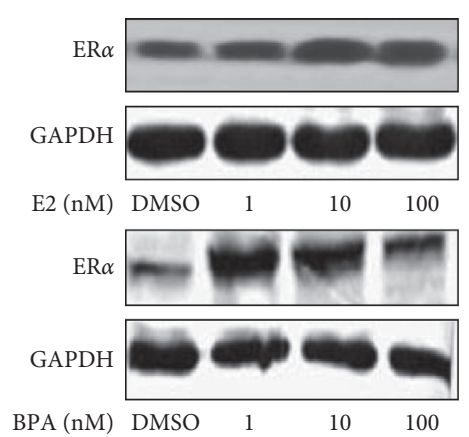

BPA (nM) $\begin{array}{llll}\text { DMSO } & 1 & 10 & 100\end{array}$
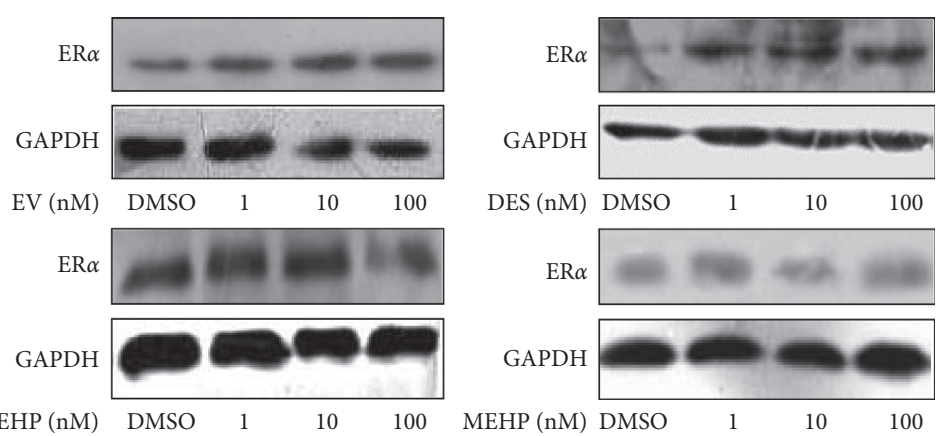

(a)
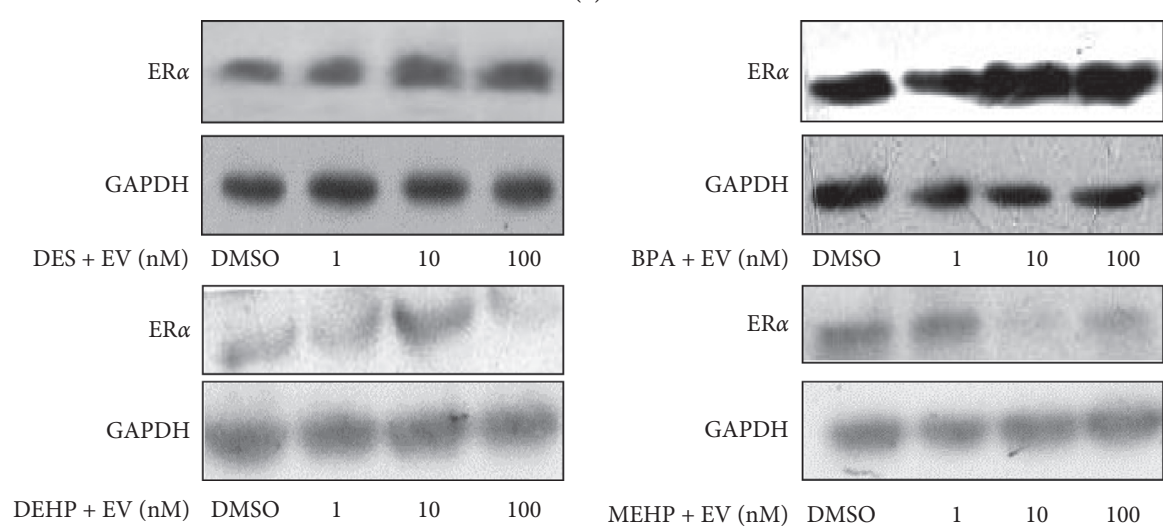

(b)

FIGURE 7: Effects of single EDCs (1-100 nM) on the expression of ER $\alpha$ by western blot analysis (a); joint effects of binary mixtures of EV and the other four EDCs (1-100 nM) on the expression of ER $\alpha$ by western blot analysis (b).

expression was analyzed by western blot. As shown in Figure 7, E2, EV, DES, and BPA upregulated the ER $\alpha$ protein expression, and the binary mixtures of EV and DES or BPA also upregulated $\mathrm{ER} \alpha$ protein expression at the exposure concentrations of 1,10 , and $100 \mathrm{nM}$.
However, single DEHP and MEHP, and the binary mixtures of EV and DEHP or MEHP cannot all upregulate $\mathrm{ER} \alpha$ protein expression, indicating that DEHP or MEHP attenuated $\mathrm{ER} \alpha$ protein expression upregulated by EV. 


\section{Discussion}

In the environment, EDCs cannot exist alone. Therefore, humans and other creatures are rarely exposed to only a single EDC, but large amounts of chemicals through a variety of ways at the same time [31]. Joint toxicity may occur when two toxic chemicals are presented in environment simultaneously [32]. When two or more pollutants act on organism, the combined effects tend to be completely different compared with a single pollutant acting on the organism $[18,33]$. Usually, joint toxicity presents four forms depending on different interactions of the two compounds. Additive effect and independent effect are the result of the two compounds having no interaction or reaction, while synergistic or antagonistic effect pertains to the interaction of the two compounds, making their toxicity increscent or reductive [34].

EV, DES, BPA, DEHP, and MEHP are typical EDCs; these high production volume chemicals could be released into the ambient environment during production, storage, and transport and coexist in environment simultaneously. Although many researches were done, they focused on single EDC or a series of EDCs at a high dosage. In these EDCs, EV, DES, and BPA have estrogenic activity, while DEHP and MEHP have antiestrogenic activity [21, 22]. Generally, estrogen compounds can induce the proliferation of estrogensensitive tumor cells and increase the intracellular ROS levels through ER $\alpha$ pathway $[25,26]$. Therefore, to evaluate the combined health risk of these different types of EDCs in environment, in this study, we investigated the effect of different types of EDCs on estrogenic activity of EV by detecting MCF-7 cell viability, intracellular ROS level change, and ER $\alpha$ protein expression. After MCF-7 cells were exposed to a series of concentration equal proportion binary mixtures of DES, BPA, DEHP and MEHP with EV for 24, 48, or $72 \mathrm{~h}$, the binary mixtures of EV and the other four EDCs can significantly attenuate cell viability induced by single $\mathrm{EV}$. The antagonistic effects of the binary mixtures of EV and the other four EDCs can be found by detecting cell viability. The joint effects of the binary mixtures of EV and the other four EDCs on intracellular ROS level change are totally different. At $24 \mathrm{~h}$, similar to the cell viability, DES and BPA decreased intracellular ROS levels induced by EV when they mixed with EV. While at 1 and $3 \mathrm{~h}$, the influence of DES, $\mathrm{BPA}$, and DEHP on intracellular ROS level change induced by EV presented inconsistent results. That is to say, at some exposure concentrations, they increased intracellular ROS levels induced by EV, while, at other exposure concentrations, they decreased intracellular ROS levels. However, in general, the joint toxicity of binary mixture of EV and the other EDCs did not interact in a synergistic fashion in inducing cell proliferation and intracellular ROS levels. Ramirez et al. [34] also found that estrogenic binary mixtures of endosulfan and dieldrin did not interact synergistically in binding to ER or activating ER-mediated responses in vitro or in vivo.

Generally, the cell proliferation of estrogen-sensitive cells can give insight into estrogenic effects of environmental contaminants [35]. ER $\alpha$ is an estrogen nuclear receptor.
Estrogen, through binding to or activating ER $\alpha$, can activate downstream signals that facilitate breast cancer cell proliferation. Both of them are generally found to be upregulated in many types of cancer and are related to cell proliferation. Numerous studies also showed that ER $\alpha$ signals are critical regulators of estrogen-sensitive tumor cell proliferation induced by environmental estrogen. For example, bisphenol F (BPF) can induce MCF-7 cells to proliferate by enhancing expression of proteins such as Cyclin D1 and Cyclin E1 via the $\mathrm{ER} \alpha$-dependent pathway [36]. Low concentration of BPA $(1 \mu \mathrm{M})$ has been shown to increase proliferation of both breast cancer cells and cancer-associated fibroblasts by an estrogen receptor-dependent pathway [37]. García et al. [38] found that environmental estrogen such as hexachlorobenzene ( $\mathrm{HCB})$ at low concentrations (5 and $50 \mathrm{nM}$ ) can increase MCF-7 cell proliferation by activating the ER $\alpha$ receptor. In the present study, we also found that single E2, $\mathrm{EV}, \mathrm{DES}$, and BPA can upregulate $\mathrm{ER} \alpha$ protein expressions increase cell viability. In addition, the binary mixtures of EV and DES or BPA also upregulated ER $\alpha$ protein expression at $24 \mathrm{~h}$, and their binary mixtures also increased cell viability at this exposure time. However, the binary mixtures of EV and DEHP or MEHP cannot increase the expression of ER $\alpha$ protein and cell viability. Some other studies also showed that DEHP and MEHP cannot activate ER $\alpha$ in estrogen receptor-positive cells $[22,39]$ and have antiestrogenic activity $[22,40]$. These results showed that ER $\alpha$ protein expression is consistent with the results of the cell viability, which indicated that these EDCs might increase proliferation of MCF-7 breast cancer cells by activating the ER $\alpha$.

However, except for ER $\alpha$, EDCs can also induce estrogen-sensitive cancer cell proliferation by estrogen member receptor $G$ protein coupled receptor 30 (GPR30) pathway [41, 42]. Moreover, there may be a crosstalk between membrane receptor such as GPR30 (nongenomic) and nuclear receptor ER (genomic) signaling pathways [43-45]. In our previous studies, we found that bisphenols such as BPF, TDP, and BPAF can induce proliferation of MCF-7 cells via interaction of ER $\alpha$ - and GPR30-mediated signaling pathways $[23,30,45]$. Therefore, the specific mechanism needs to further study more proteins associated with the cell proliferation such as GPR30 and its downstream signals.

Occurrence of ROS in cells has been a common response of chemical toxicity, which can damage active cellular substance, such as DNA, lipids, and proteins [46]. The redox system may be especially important during estrogen-induced cell proliferation, and different cellular signals may operate in response to varying levels of estrogen-induced ROS [47]. Many studies have found that natural or synthetic estrogen can induce ROS generation in breast cancer cells by estrogen receptor pathway, and increased ROS has been involved in both cell proliferation and carcinogenesis [25-27, 47]. However, cells have various antioxidant defense systems, consisting of both enzymatic and nonenzymatic substances, to reduce ROS levels and thus protect the cells from ROS damage [46]. When the balance between ROS and antioxidant defense system is broken, oxidative damage, or so-called oxidative stress, occurs. However, the balance 
between ROS and antioxidant defense system is a dynamic process. The levels of generated ROS in intracellular are closely related to exposure concentrations and exposure time of the exposed chemicals [48]. In this study, we also found that, at different exposure concentrations and different exposure time, the intracellular ROS levels are different. The ROS generation in the binary mixtures of EV and the other four EDCs also presented different change trends at different exposure concentrations and different exposure time. The deeper reasons need further study.

\section{Conclusions}

Single EV, DES, and BPA can promote the proliferation of MCF-7 cells, and EV has the strongest potency in inducing cell proliferation. Cell viability induced by EV was significantly attenuated by DES, BPA, DEHP, and MEHP when they were mixed with EV. ER $\alpha$ signals play a key role in MCF-7 cell proliferation induced by low-concentration EDCs like EV, DES, or BPA. Single EV, BPA, DES, DEHP, and MEHP elevated intracellular ROS levels for different exposure durations. DES and BPA decreased intracellular ROS levels induced by EV when they mixed with EV for $24 \mathrm{~h}$. These results indicated that the joint effects of binary mixtures of EV and other EDCs do not interact synergistically in inducing cell proliferation, intracellular ROS generation, and ER $\alpha$ protein expression. The results have important implications in the human risk assessments of EV coexposed with other EDCs in the environment.

\section{Data Availability}

The data used to support the findings of this study are included within the article.

\section{Conflicts of Interest}

The authors declare that there are no conflicts of interest regarding the publication of this paper.

\section{Acknowledgments}

The present work was supported by the National Natural Science Foundation of China (nos. 21777093 and 21507078), Open Foundation of Guangdong Key Laboratory of Environmental Catalysis and Health Risk Control (GKECHRC02), and the Program for Changjiang Scholars and Innovative Research Team in University (no. IRT13078).

\section{References}

[1] P. A. Fowler, M. Bellingham, K. D. Sinclair et al., "Impact of endocrine-disrupting compounds (EDCs) on female reproductive health," Molecular and Cellular Endocrinology, vol. 355, no. 2, pp. 231-239, 2012.

[2] R. Lauretta, A. Sansone, M. Sansone, F. Romanelli, and M. Appetecchia, "Endocrine disrupting chemicals: effects on endocrine glands," Frontiers in Endocrinology, vol. 10, 2019.

[3] L. M. Borgelt and C. W. Martell, "Estradiol valerate/dienogest: a novel combined oral contraceptive," Clinical Therapeutics, vol. 34, no. 1, pp. 37-55, 2012.
[4] A. M. Andersson and N. E. Skakkebaek, "Exposure to exogenous estrogens in food: possible impact on human development and health," European Journal of Endocrinology, vol. 140, no. 6, pp. 477-485, 1999.

[5] G. Senol, Y. Mecit, G. Kenan, and K. Turan, "Effects of oral administration of estradiol valerate on gonadal sex differentiation in the rainbow trout, Oncorhynchus mykiss," Journal of Animal and Veterinary Advances, vol. 7, no. 11, pp. 1400-1404, 2008.

[6] Z. Chu, Y. Wu, S. Gong et al., "Effects of estradiol valerate on steroid hormones and sex reversal of female rice field eel, Monopterus albus (Zuiew)," Journal of the World Aquaculture Society, vol. 42, no. 1, pp. 96-104, 2011.

[7] B. Lei, J. Kang, Y. Yu, J. Zha, W. Li, and Z. Wang, " $\beta$-estradiol 17-valerate affects embryonic development and sexual differentiation in Japanese medaka (Oryzias latipes)," Aquatic Toxicology, vol. 134-135, pp. 128-134, 2013.

[8] B. Lei, S. Huang, Y. Zhou, D. Wang, and Z. Wang, "Levels of six estrogens in water and sediment from three rivers in Tianjin area, China," Chemosphere, vol. 76, no. 1, pp. 36-42, 2009.

[9] W. Jiang, Y. Yan, M. Ma et al., “Assessment of source water contamination by estrogenic disrupting compounds in China," Journal of Environmental Sciences, vol. 24, no. 2, pp. 320-328, 2012.

[10] A. L. Herbst, H. Ulfelder, and D. C. Poskanzer, "Adenocarcinoma of the vagina-association of maternal stilbestrol therapy with tumor appearance in young women," American Journal of Obstetrics and Gynecology, vol. 181, no. 6, 1574-1575, 1999.

[11] O. B. Adedeji, E. J. Durhan, N. Garcia-Reyero et al., "Shortterm study investigating the estrogenic potency of diethylstilbesterol in the fathead minnow (Pimephales promelas)," Environmental Science \& Technology, vol. 46, no. 14, pp. 7826-7835, 2012.

[12] D. J. Caldwell, F. Mastrocco, T. H. Hutchinson et al., "Derivation of an aquatic predicted no-effect concentration for the synthetic hormone, $17 \alpha$-ethinyl estradiol," Environmental Science \& Technology, vol. 42, no. 19, pp. 7046-7054, 2008.

[13] G. Pojana, A. Gomiero, N. Jonkers, and A. Marcomini, "Natural and synthetic endocrine disrupting compounds (EDCs) in water, sediment and biota of a coastal lagoon," Environment International, vol. 33, no. 7, pp. 929-936, 2007.

[14] T.-S. Chen, T.-C. Chen, K.-J. C. Yeh et al., "High estrogen concentrations in receiving river discharge from a concentrated livestock feedlot," Science of the Total Environment, vol. 408, no. 16, pp. 3223-3230, 2010.

[15] L. Wang, G.-G. Ying, J.-L. Zhao et al., "Assessing estrogenic activity in surface water and sediment of the Liao River system in Northeast China using combined chemical and biological tools," Environmental Pollution, vol. 159, no. 1, pp. 148-156, 2011.

[16] R. Guedes-Alons, Z. Sosa-Frrera, and J. Juan SantanaRodríguez, "Simultaneous determination of hormonal residues in treated waters using ultrahigh performance liquid chromatography-tandem mass spectrometry," Journal of Analytical Methods in Chemistry, vol. 2013, Article ID 210653, 8 pages, 2013.

[17] Y. Q. Huang, C. K. C. Wong, J. S. Zheng et al., "Bisphenol A (BPA) in China: a review of sources, environmental levels, and potential human health impacts," Environment International, vol. 42, pp. 91-99, 2012.

[18] B. Huang, M. Feng, D. Li, and Y. Yang, "Antagonistic joint toxicity assessment of two current-use phthalates with waterborne copper in liver of Carassius auratus using 
biochemical biomarkers," Ecotoxicology and Environmental Safety, vol. 116, pp. 107-112, 2015.

[19] X. Wu, H. Hong, X. Liu et al., "Graphene-dispersive solidphase extraction of phthalate acid esters from environmental water," Science of The Total Environment, vol. 444, pp. 224230, 2013.

[20] J. C. Caldwell, "DEHP: genotoxicity and potential carcinogenic mechanisms-a review," Mutation Research/Reviews in Mutation Research, vol. 751, no. 2, pp. 82-157, 2012.

[21] R. Czernych, M. Chraniuk, P. Zagożdżon, and L. Wolska, "Characterization of estrogenic and androgenic activity of phthalates by the XenoScreen YES/YAS in vitro assay," Environmental Toxicology and Pharmacology, vol. 53, pp. 95104, 2017.

[22] D.-H. Kim, C. G. Park, S. H. Kim, and Y. J. Kim, "The effects of mono-(2-ethylhexyl) phthalate (MEHP) on human estrogen receptor (hER) and androgen receptor (hAR) by YES/YAS in vitro assay," Molecules, vol. 24, no. 8, p. 1558, 2019.

[23] B. Lei, S. Sun, C. Feng et al., "Bisphenol AF exerts estrogenic activity in MCF-7 cells through activation of Erk and PI3K/ Akt signals via GPER signaling pathway," Chemosphere, vol. 220, pp. 362-370, 2019.

[24] S.-L. Liu, X. Lin, D.-Y. Shi, J. Cheng, C.-Q. Wu, and Y.-D. Zhang, "Reactive oxygen species stimulated human hepatoma cell proliferation via cross-talk between PI3-K/PKB and JNK signaling pathways," Archives of Biochemistry and Biophysics, vol. 406, no. 2, pp. 173-182, 2002.

[25] P. K. S. Mahalingaiah and K. P. Singh, "Chronic oxidative stress increase growth and tumorigenic potential of MCF-7 breast cancer cells," PLoS One, vol. 9, no. 1, Article ID e87371, 2014.

[26] K. Lata and T. K. Mukherjee, "Knockdown of receptor for advanced glycation end products attenuate $17 \alpha$-ethinyl-estradiol dependent proliferation and survival of MCF-7 breast cancer cells," Biochimica et Biophysica Acta (BBA)-General Subjects, vol. 1840, no. 3, pp. 1083-1091, 2014.

[27] J. Sastre-Serra, A. Valle, M. M. Company, I. Garau, J. Oliver, and P. Roca, "Estrogen down-regulates uncoupling proteins and increases oxidative stress in breast cancer," Free Radical Biology and Medicine, vol. 48, no. 4, pp. 506-512, 2010.

[28] K. Lin, "Joint acute toxicity of tributyl phosphate and triphenyl phosphate to Daphnia magna," Environmental Chemistry Letters, vol. 7, no. 4, pp. 309-312, 2009.

[29] Y. N. Gao, B. Y. Liu, F. J. Ge et al., "Joint effects of allelochemical nonanoic acid, $N$-phenyl-1-naphtylamine and caffeic acid on the growth of Microcystis aeruginosa," Allelopathy Journal, vol. 35, no. 2, pp. 249-257, 2015.

[30] B. Lei, W. Peng, G. Xu et al., "Activation of G protein-coupled receptor 30 by thiodiphenol promotes proliferation of estrogen receptor $\alpha$-positive breast cancer cells," Chemosphere, vol. 169, pp. 204-211, 2017.

[31] N. Lazarevic, A. G. Barnett, P. D. Sly, and L. D. Knibbs, "Statistical methodology in studies of prenatal exposure to mixtures of endocrine-disrupting chemicals: a review of existing approaches and new alternatives," Environmental Health Perspectives, vol. 127, no. 2, Article ID 026001, 2019.

[32] N. Liu, Y. Wang, F. Ge, S. Liu, and H. Xiao, "Antagonistic effect of nano- $\mathrm{ZnO}$ and cetyltrimethyl ammonium chloride on the growth of Chlorella vulgaris: dissolution and accumulation of nano-ZnO," Chemosphere, vol. 196, pp. 566-574, 2018.

[33] L. Mignuez, R. Bureau, and M.-P. Halm-Lemeille, "Joint effects of nine antidepressants on Raphidocelis subcapitata and
Skeletonema marioi: a matter of amine functional groups," Aquatic Toxicology, vol. 196, pp. 117-123, 2018.

[34] T. Ramirez, A. Buechse, M. Dammann, S. Melching-Kollmuß, C. Woitkowiak, and B. Van Ravenzwaay, "Effects of estrogenic binary mixtures in the yeast estrogen screen (YES)," Regulatory Toxicology and Pharmacology, vol. 70, no. 1, pp. 286-296, 2014.

[35] M. Shim, J.-Y. Bae, Y. J. Lee, and M.-J. Ahn, "Tectoridin from Maackia amurensis modulates both estrogen and thyroid receptors," Phytomedicine, vol. 21, no. 5, pp. 602-606, 2014.

[36] J.-Y. Kim, H.-G. Choi, H.-M. Lee, G.-A. Lee, K.-A. Hwang, and K.-C. Choi, "Effects of bisphenol compounds on the growth and epithelial mesenchymal transition of MCF-7 CV human breast cancer cells," Journal of Biomedical Materials Research, vol. 31, no. 4, pp. 358-369, 2017.

[37] M. Pupo, A. Pisano, R. Lappano et al., "Bisphenol A induces gene expression changes and proliferative effects through GPER in breast cancer cells and cancer-associated fibroblasts," Environmental Health Perspectives, vol. 120, no. 8, pp. 1177-1182, 2012.

[38] M. A. García, D. Peña, L. Álvarez et al., "Hexachlorobenzene induces cell proliferation and IGF-I signaling pathway in an estrogen receptor $\alpha$-dependent manner in MCF-7 breast cancer cell line," Toxicology Letters, vol. 192, no. 2, pp. 195-205, 2010.

[39] C. Park, J. Lee, B. Kong et al., "The effects of bisphenol A, benzyl butyl phthalate, and di (2-ethylhexyl) phthalate on estrogen receptor alpha in estrogen receptor-positive cells under hypoxia," Environmental Pollution, vol. 248, pp. 774781, 2019.

[40] H. Yu, D. J. Caldwell, and R. P. Suri, "In vitro estrogenic activity of representative endocrine disrupting chemicals mixtures at environmentally relevant concentrations," Chemosphere, vol. 215, pp. 396-403, 2019.

[41] M. Barton, E. J. Filardo, S. J. Lolait, P. Thomas, M. Maggiolini, and E. R. Prossnitz, "Twenty years of the G protein-coupled estrogen receptor GPER: historical and personal perspectives," The Journal of Steroid Biochemistry and Molecular Biology, vol. 176, pp. 4-15, 2018.

[42] L.-Y. Cao, X.-M. Ren, C.-H. Li et al., "Bisphenol AF and bisphenol $B$ exert higher estrogenic effects than bisphenol A via G protein-coupled estrogen receptor pathway," Environmental Science \& Technology, vol. 51, no. 19, pp. 1142311430, 2017.

[43] Z.-G. Sheng and B.-Z. Zhu, "Low concentrations of bisphenol A induce mouse spermatogonial cell proliferation by $G$ protein-coupled receptor 30 and estrogen receptor- $\alpha$," Environmental Health Perspectives, vol. 119, no. 12, pp. 17751780, 2011.

[44] L.-C. Ge, Z.-J. Chen, H.-Y. Liu et al., "Involvement of activating ERK1/2 through $G$ protein coupled receptor 30 and estrogen receptor $\alpha / \beta$ in low doses of bisphenol A promoting growth of sertoli TM4 cells," Toxicology Letters, vol. 226, no. 1, pp. 81-89, 2014.

[45] B. Lei, Y. Huang, Y. Liu et al., "Low-concentration BPF induced cell biological responses by the ER $\alpha$ and GPER1-mediated signaling pathways in MCF-7 breast cancer cells," Ecotoxicology and Environmental Safety, vol. 165, pp. 144-152, 2018.

[46] Z. Ji, Y. Zhang, J. Tian, F. Wang, M. Song, and H. Li, “Oxidative stress and cytotoxicity induced by tetrachlorobisphenol A in Saccharomyces cerevisiae cells," Ecotoxicology and Environmental Safety, vol. 161, pp. 1-7, 2018. 
[47] L. Yuan, A. K. Dietrich, and A. M. Nardulli, " $17 \beta$-estradiol alters oxidative stress response protein expression and oxidative damage in the uterus," Molecular and Cellular Endocrinology, vol. 382, no. 1, pp. 218-226, 2014.

[48] B. Lei, S. Sun, J. Xu et al., "Low-concentration BPAF-and BPFinduced cell biological effects are mediated by ROS in MCF-7 breast cancer cells," Environmental Science and Pollution Research, vol. 25, no. 4, pp. 3200-3208, 2018. 